\title{
Automated analysis of invadopodia dynamics in live cells
}

Multiple cell types form specialized protein complexes that are used by the cell to actively degrade the surrounding extracellular matrix. These structures are called podosomes or invadopodia and collectively referred to as invadosomes. Due to their potential importance in both healthy physiology as well as in pathological conditions such as cancer, the characterization of these structures has been of increasing interest. Following early descriptions of invadopodia, assays were developed which labelled the matrix underneath metastatic cancer cells allowing for the assessment of invadopodia activity in motile cells. However, characterization of invadopodia using these methods has traditionally been done manually with time-consuming and potentially biased quantification methods, limiting the number of experiments and the quantity of data that can be analysed. We have developed a system to automate the segmentation, tracking and quantification of invadopodia in timelapse fluorescence image sets at both the single invadopodia level and whole cell level. We rigorously tested the ability of the method to detect changes in invadopodia formation and dynamics through the use of well-characterized small molecule inhibitors, with known effects on invadopodia. Our results demonstrate the ability of this analysis method to quantify changes in invadopodia formation from live cell imaging data in a high throughput, automated manner. 
4 1. UNC/NCSU Joint Department of Biomedical Engineering, University of North Carolina at 5 Chapel Hill, Chapel Hill, NC

6 2. Department of Cell Biology and Physiology, University of North Carolina at Chapel Hill, 7 Chapel Hill, NC

8 3. Lineberger Comprehensive Cancer Center, University of North Carolina at Chapel Hill, Chapel 9 Hill, NC

10 4. Howard Hughes Medical Institute, Chevy Chase, MD

11 5. Department of Computer Science, University of North Carolina at Chapel Hill, Chapel Hill, $12 \mathrm{NC}$

13 6. Department of Pharmacology, University of North Carolina at Chapel Hill, Chapel Hill, NC

14 \# Current Addresses:

15 MEB: Department of Biomedical Engineering, Duke University. 101 Science Drive, Durham NC 16 SJC: Monash Institute of Pharmaceutical Sciences, Monash University, Parkville VIC Australia

$17 *$ Address Correspondence To:

18 Shawn M. Gomez. Tel: +1 919966 4959. Email: $\underline{\text { smgomez@unc.edu }}$

19 or

20 James E. Bear. Tel: +1 9199665241 Email: jbear@email.unc.edu

21 Abbreviations: DMSO; Dimethyl Sulfoxide, ECM; extracellular matrix, FAK; Focal Adhesion 22 Kinase, PBS; Phosphate Buffered Saline, MMPs; matrix metalloproteinases

\section{Introduction}

24 Migration through a three dimensional environment, such as during embryonic development or 25 metastasis, is a multistage process beginning with the migration of either single cells or groups of 
cells away from the primary site and into the surrounding ECM (Extra Cellular Matrix). To accomplish this migration, the ECM is commonly degraded, typically through the use of matrix metalloproteinases (MMPs) to form paths through which the cells can move. Invadopodia and podosomes, collectively termed invadosomes, are the two structures most commonly associated with this behavior. In the case of cancer cells and the process of metastasis, the formation of invadopodia that actively degrade the ECM is a common observation (Destaing et al. 2011; Murphy \& Courtneidge 2011). Importantly, while markers such as cortactin and Arp2/3 subunits can help discern these structures from other actin-rich structures, the direct observation of degradation activity is required in order to accurately identify these invasive structures.

Invadopodia were first imaged in Rous sarcoma virus transformed cells using several imaging methodologies including interference reflection and fixed cell labelling (Chen 1989; Tarone et al. 1985). To measure degradation activity in vitro, quantitative imaging assays of invadopodia behavior have been developed that use fluorescently labeled ECM to visualize regions of degradation caused by invadopodia (Artym et al. 2009). By combining fluorescently labeled ECM and fluorescently labelled intracellular markers of invadopodia, such as cortactin (Artym et al. 2006), cofilin (Stoletov et al. 2013) or actin (Albiges-Rizo et al. 2009), the activity of single invadopodia can be followed through time (Sharma et al. 2013a). The continued development of methods to observe invadopodia formation and ECM degradation has made it possible to quantify the effect of siRNA knockdown or drug treatment on invadopodia dynamics (Beaty et al. 2013; Sharma et al. 2013b). However, the time-lapse image sets produced using these methods have traditionally been analysed using manual selection/analysis methods that are time-consuming and potentially biased in the selection of which invadopodia will be measured.

In order to improve the reliability of invadopodia measurement systems, we have developed a system to automate the segmentation, tracking and quantification of invadopodia in time-lapse fluorescence image sets at both the single invadopodia level and whole cell level. These methods use the fluorescently labeled ECM images to determine when and where individual invadopodia 52 or cells are degrading the matrix, making detailed studies of invadopodia formation, timing and activity possible. Since these methods were initially developed using LifeAct-GFP as marker of

54 invadopodia, the system does not assume that all bright puncta will become invadopodia and 55 degrade the ECM and instead uses the changes in the underlying ECM to classify puncta. The 56 first set of tools has been designed to identify and track single invadopodia and the corresponding 
57 ECM, as might be studied using images taken at $60 \mathrm{X}$ magnification. The second set of tools does

58 not attempt to track individual invadopodia and instead focuses on cell population images which

59 could be taken at 20X magnification. We have made both sets of tools available as open source

60 packages and made a web-based version of the single invadopodia analysis available.

\section{Materials and Methods}

62 Reagents

63 All chemicals and reagents were purchased from Sigma (St Louis, MO) unless otherwise stated.

64 The BB94, Purvalanol A, FAK Inhibitor II and PP2 compounds were all purchased from EMD

65 BioSciences (La Jolla, CA). All drugs were soluble in DMSO, which was used as a control for all 66 experiments. The BB94 and FAK Inhibitor II were used at a final concentration of $5 \mu \mathrm{M}$, while 67 Purvalanol A was used at $2 \mu \mathrm{M}$ and PP2 was used at $10 \mu \mathrm{M}$. The Alexa Fluor 568 protein 68 labeling kit used to label the gelatin matrix was purchased from Invitrogen (Eugene, OR), as were 69 all cell culture reagents. Fugene 6 used for transfections was purchased from Roche Diagnostics 70 (Indianapolis, IN). For dynamic visualization of actin, the LifeAct peptide (Riedl et al. 2008) was 71 cloned into the previously described pLL 5.0 GFP Lenti-viral base vector (Wang \& McNiven $72 \underline{2012})$.

\section{3}

74

75

76

77

78

79

80

81

82 To prepare ECM substrate for the invadopodia assays, 0.2\% Type A gelatin from porcine skin 83 was fluorescently labeled using the Alexa Fluor 568 protein labeling kit according to the

84 manufacturer instructions. Culture vessels were coated at room temperature with thin layers of $8520 \%$ poly-L-lysine for 20 mins followed by $0.5 \%$ glutaraldehyde (Electron Microscopy Sciences,

\section{Cell Lines and Culture}

Wild Type and LifeAct-GFP WM2664 cell lines and 293FT Hek cells were maintained in DMEM with $10 \% \mathrm{FBS}$ and $1 \% \mathrm{PSG}$ at $37^{\circ} \mathrm{C}$ in a humidified atmosphere with $5 \% \mathrm{CO}_{2}$. Lentiviral production was performed as previously described in (Wang \& McNiven 2012). Briefly, lentiviral expression plasmids were transfected into 293FT cells with the construct of interest using Fugene6. After $24 \mathrm{hrs}$, media is changed and viral media is applied to $30-50 \%$ confluent WM2664 cells in a 6-well dish for 48hrs. Fluorescent cells were FACS sorted for the $20-80 \%$ range of GFP positive cells.

\section{Fluorescent Gelatin Matrix}


86 Hatfield, PA) for 15 mins. An 8:1 ratio of unlabeled to labeled gelatin was prepared and incubated

87 on culture vessels for 10 mins. Following gelatin incubation a $5 \mathrm{mg} / \mathrm{mL}$ solution of $\mathrm{NaBH}_{4}$

88 (Fisher Scientific, Fair Lawn, NJ) was incubated for 15 mins to quench any remaining 89 glutaraldehyde. Between each level of coating, the dishes were washed three times with 90 phosphate buffered saline (PBS).

\section{Single Invadopodia Assay Imaging Parameters}

92 LifeAct-GFP WM2664 cells were plated (+/- inhibitors) onto heated bioptech dishes (Bioptech 93 Inc., Butler, PA) coated with Alexa Fluor 568 labeled gelatin, as described above. Cells were 94 allowed to equilibrate on the microscope $1 \mathrm{hr}$ before imaging. Random fields of view were 95 selected and time-lapse microscopy was performed on an Olympus IX- 81 inverted microscope (60x, 1.3NA objective) with a Hamamatsu CCD camera (model c4742-80-12AG) and a Prior Lumen200Pro epifluorescence system (Olympus America, Center Valley, PA) on a heated stage with heated lid receiving a constant stream of humidified $5 \% \mathrm{CO}_{2}$ to maintain cell viability. Images were captured in both the 488 and 568 channels every 5 mins for 12 hrs using MetaMorph Imaging software (Molecular Devices, Sunnyvale, CA).

101

102

103

104

105

106

107

108

109

110

111

112

113

114

115

\section{Cell Population Assay Imaging Parameters}

LifeAct-GFP WM2664 cells (+/- inhibitors) were plated onto MatTek $35 \mathrm{~mm}$ glass bottom dishes (MatTek Corporation, Ashland, MA) coated with Alexa Flour 568 labeled gelatin, as described above. Cells were allowed to equilibrate in the incubation chamber of the microscope, heated to $37^{\circ} \mathrm{C}$ with a humidified atmosphere of $5 \% \mathrm{CO}_{2}$, for $1 \mathrm{hr}$ before imaging. Cells were imaged in the Olympus VivaView microscope with a 20x/0.75NA objective and morotized magnification changer set to $1 \mathrm{x}$, using a Hamamatsu Orca R2 cooled CCD camera (Olympus America, Center Valley, PA). A template was used to select 25 predetermined positions in each dish and images in the 488 and 568 channels were captured every 30 mins for $25 \mathrm{hrs}$.

\section{Invadopodia Analysis}

Before analyzing, the fluorescent ECM images were photobleach corrected. To ensure that the degradation of ECM was not incorrectly identified as photobleaching, only pixels outside the cell bodies were considered when photobleaching correction was applied. Images were then flat-field corrected and the single cell time-lapse images for single cell assays were registered (the cell population movies did not require registration). In the ECM images used for the single 
116 invadopodia analysis, the average fluorescence outside the cell bodies was set to 1000 to allow

117 the local difference values to be compared between ECM preparations. The LifeAct-GFP images

118 for the single invadopodia analysis were not pre-processed. The LifeAct-GFP images for the

119 population analysis were flat-field corrected. The analysis software uses a high-pass filter and

120 threshold to identify regions of high actin concentration (potential invadopodia) that co-localize

121 with underlying ECM degradation to identify active invadopodia and track them through time.

\section{Results}

123 The results section is divided into two parts, the first of which deals with the analysis and 124 quantification of single Invadopodia in $60 \mathrm{X}$ images. The second portion of the results details the 125 analysis and quantification of ECM degradation by whole cells.

\section{Identification and Tracking of LifeAct-GFP Puncta in 60X Images}

127 The development of software and subsequent analysis of invadopodia was performed in multiple 128 steps. The first step of analysis involved the development of methods to segment and track single 129 actin aggregates, termed puncta, from images of LifeAct-GFP. After identifying actin puncta, a 130 set of measurements were made from the fluorescent ECM images at each time point to classify 131 the effect of the puncta on the underlying ECM. As only a portion of the population of LifeAct132 GFP puncta degrade the matrix, puncta that degrade the matrix are classified as invadopodia. 133 Additional collected properties such as degradation rates were then used in further analyses as 134 described below.

135 To identify LifeAct-GFP labelled puncta in WM2664 cells (Figure 1A), untreated and BB94 136 treated cells were imaged by time-lapse microscopy. In order to ensure that the automated system 137 would have decision thresholds similar to that of experts doing a manual analysis, prior to 138 automated analysis, images assessed by three experienced independent observers who manually 139 identified and segmented LifeAct-positive puncta. A final consensus segmentation was reached 140 by majority vote from the individual manual segmentations. These invadopodia segmentations, 141 determined by our observers where then used to test potential segmentation strategies, threshold 142 settings and determine appropriate filters. 
143 The first stage of the automated segmentation pipeline used a high-pass filter to remove the

144 background noise from the LifeAct-GFP signal (Figure 1B) and then determined the mean and 145 standard deviation of the high-pass filtered pixel intensities for use as thresholds. To identify 146 individual puncta, a seed-based region-growing segmentation method was used. To identify seed 147 pixels in each image, intensity thresholds from 1-10 standard deviations were tested and these 148 automatically segmented regions were compared to those identified through manual segmentation 149 (Figure 1C), with the false positive and negative rates computed as previously described ( $\underline{\mathrm{Matov}}$ 150 et al. 2010). As the seed threshold increased, the rate of false positives decreased, while the rate 151 of false negatives increased. We also tested minimum seed sizes, ranging from 2-10 pixels, and 152 observed the same general behavior in the false positive and false negative rates as seed size 153 increased. To balance these factors, we empirically selected a standard deviation threshold of 3 154 and a minimum size of 6 pixels to identify the puncta seeds (Figure 1D).

155 After identification of the puncta seeds, a second threshold was selected to expand around each of 156 the identified seed regions. To assess the performance of the seed expansion procedure, we 157 measured the degree of overlap between the manually segmented puncta and the matching 158 computer segmented puncta. We tested thresholds from 0-3 standard deviations from the mean 159 (Figure 1E). As expected, as the seed expansion threshold increases, the false positive rate 160 decreases, while the false negative rate increases. We selected a seed expansion threshold of 1.75 161 to balance these two factors. We note that thresholds may need to adjusted depending on the 162 fluorescent marker or cell line used. As a final filter, we also considered the area and ratio 163 between the major and minor axes of the segmented puncta. To determine these filters, we 164 measured these properties in the manually identified puncta (Figure 1F). We used the minimum 165 and maximum values for the area and the major over minus axes ratio as filters for any objects 166 identified after seed identification and expansion. The cell edge was also identified in the 167 LifeAct-GFP signal using a previously published method (Hoshino et al. 2013). The properties 168 for each identified puncta (Figure $1 \mathrm{G}$ ) were then collected, which included area and the distance 169 of the puncta from the nearest cell edge.

170 Each puncta was also tracked through the experiment, using overlap in adjacent frames to 171 connect the segmented puncta. The majority of the identified puncta were present for only one 172 frame (Figure 2A), but a population of puncta that could be followed for 12 frames or more 173 (Figure 2A inset) was also observed. For puncta that live for 12 frames or more, the average area 
174 (Figure 2B) and the average distance from the nearest cell edge (Figure 2C) were calculated over 175 time.

\section{Determination of ECM Degradation by Puncta}

177 As described previously, degradation of the matrix is a necessary condition for an individual 178 puncta to be classified as a functional invadopodia. This degradation is visible as dark regions 179 that develop in a fluorescently labelled gelatin matrix underneath puncta over time. In order to 180 detect this change in the ECM, the average ECM intensity immediately underneath each puncta 181 and the area in a five-pixel border surrounding the puncta was tracked over time (Figure 3A). 182 Areas within the surrounding border occupied by another identified puncta were excluded from 183 quantitation. The difference between the average intensity in the surrounding ECM and the ECM 184 underneath each puncta was used to calculate the local fluorescence difference, so that puncta that have degraded the ECM will have positive values in the local fluorescence difference, while non-degrading puncta will have values near zero. To account for irregularities in the gelatin matrix, the intensity of the matrix before the puncta appeared in the time-lapse image set was also determined. This pre-birth local fluorescence difference was calculated over the same pixels using the image immediately before the appearance of puncta. In cases where the puncta is present at the beginning of the time-lapse, the first image of the ECM time-lapse was used. The pre-birth local difference calculated at each image was used to correct the observed local intensity difference, giving the corrected local intensity difference.

193 To classify the puncta as active invadopodia, the values of the local difference, pre-birth local 194 difference and the corrected local difference were analysed. To ensure that sufficient data from 195 each puncta was assessed, we limited our search to only those puncta present in the time-lapses

196 for at least one hour (12 images under our experimental protocol). Application of this filtering 197 constraint left 2,323 untreated puncta and 979 DMSO, 294 BB94, 533 Focal Adhesion Kinase (FAK) inhibitor, 83 PP2 and 125 Purvalanol A treated puncta. For invadopodia, we would expect the local difference and corrected local difference values to be positive (puncta that do not degrade the matrix; see a sample invadopodia in Figure 3B) and for both of these values to average around zero or negative for non-invadopodia puncta (see a sample non-invadopodia puncta in Figure 3C). Next, we tested whether the mean local intensity difference and the mean corrected local intensity difference were statistically different from zero using a t-test. After applying a Bonferroni correction for the number of tests run, 336 untreated, 44 control, 12 BB94 
treated and 35 FAK inhibitor treated puncta were classified as invadopodia. As expected, no actively degrading invadopodia were detected in the PP2 or Purvalanol A treated cells. The mean local corrected difference was greater in the untreated, control and FAK inhibitor treated cells as compared to the BB94 treated cells (Figure 3D). The calculated differences in invadopodia number following drug treatment correlate with previously identified outcomes on invadopodia for each inhibitor tested (Chan et al. 2009; Hoshino et al. 2012; Wang et al. 1994) and demonstrate the accuracy of the automated detection methods developed here. The 7 invadopodia identified in BB94 treated cells were manually identified as false positives and we excluded them from further analysis. The low number of false positives identified in the BB94 treated cells further demonstrates the improved performance capable through the use of these automated imaging methods.

\section{Measurement of Invadopodia Properties}

Invadopodia identified in the untreated, control and FAK treated invadopodia were used to measure the mean area of each invadopodia and average distance of invadopodia to the nearest cell edge (Figure 4A, B). No differences were detected in the average area of invadopodia between conditions (Figure 4A). The average distance of invadopodia from the edge of the cell was decreased by $28 \%$ in control cells compared to untreated cells and by $38 \%$ after treatment with FAK inhibitor (Figure 4B). There was no difference observed in invadopodia distance from the edge between control cells and FAK inhibitor treated cells (Figure 4B). The lifetime of invadopodia showed increases of $45 \%$ as compared to the untreated cells following treatment with the FAK inhibitor (Figure 4C).

The amount of time from puncta formation until maximum degradation was also quantified. To measure this property a smoothed curve was fit to the degradation curve of each invadopodia (e.g. the curve shown in Figure 3B). The earliest time point at which the smoothed values hit $90 \%$ of the maximum was chosen as the time to maximum degradation. The time to reach maximum degradation was increased by $77 \%$ in the FAK treated cells when compared with the untreated cells (Figure 4D).

\section{Quantification of Whole-Cell Degradation Behaviors}

233 To complement the single invadopodia analysis, a system for quantifying the ECM degradation

234 capacity of entire cell populations was also developed. As with the individual invadopodia work, 
235 WM2664 cells expressing LifeAct-GFP and exposed to the same drug treatments used for single 236 cell analysis were used to test the capabilities of this system. After image pre-processing, cells in 237 the LifeAct-GFP images (Figure 5A) were identified using the same algorithm as that used to 238 find the cell outline in the single invadopodia analysis (Figure 5B). To minimize the number of 239 cell clusters found and make sure results describe measurements for individual cells, we 240 quantified the areas of all observed single cells and multi-cell clusters (Figure 5C). We 241 empirically set a minimum size threshold of 1,500 pixels $\left(107 \mu^{2}\right)$ to exclude small debris and a 242 maximum size threshold of 20,000 pixels $\left(1422 \mu \mathrm{m}^{2}\right)$ to exclude cell clusters from further 243 analysis.

244 By identifying and tracking individual cells through time, we observed a bimodal distribution of 245 cell lifetimes (Figure 5D). Objects present for less than 10 hours were often non-adhered cell 246 bodies or other debris in the field of view and were excluded from further analysis. In parallel 247 with the cell images, images were also taken of the fluorescent ECM (Figure 5E). Using the 248 segmented cells and the intensity of the underlying matrix, we were able to observe both cells 249 that degraded the matrix as well as those that did not (Figure 5F).

\section{Determination of Cellular ECM Degradation by Whole Cells}

251 The segmented cell and ECM images (Figure 5) were used to classify cells as either degraders or 252 non-degraders. To accomplish this, methods similar to the analysis of single invadopodia were 253 used (Figure 6A). More specifically, for cells identified in each image, the average ECM intensity 254 underneath and within a 40 pixel border around each cell was measured, while excluding any 255 region overlapping with another cell and the corresponding regions in the prior ECM image. The 256 average change in fluorescence intensity from the prior image to current image and in the 257 surrounding 40 pixel border was then calculated. To allow these values to be compared across 258 differing ECM intensity regions, the values were saved as the percentage difference between the 259 ECM intensity underneath each cell and the surrounding region. Therefore, invading cells (i.e. 260 those degrading the matrix) would be expected to have lower ECM intensities immediately 261 underneath the cell. We then collected time-series images for each cell present for at least ten 262 hours (example time-series is shown in Figure 6B).

263 In addition to the image-by-image assessment of the percentage of the ECM degraded, the overall 264 percentage of fluorescent ECM degraded by each cell was also assessed (Figure 6C). To find the 
overall degradation percentage for each cell, the area of influence for each cell was determined by finding the amount of time the cell covered each pixel location in the field of view. Any pixel location covered for at least $2.5 \mathrm{hrs}$ was considered to be in that cell's area of influence. The change in fluorescence intensity from the first image of the ECM time-lapse and the area surrounding the cell was then calculated in the same manner as when calculating the percentage change from image to image.

Each cell was classified as degrading or non-degrading on a per image basis using the between images and total ECM degradation percentages calculated. As the BB94-treatment is expected to block all activity of the MMPs, degradation percentages found in the BB94 treated cells were used as a negative control. We empirically assessed the potential cut-off values to minimize the number of false positives (ie: the number of BB94-treated cells classified as degraders).

\section{Measurement of Matrix Degrading Behaviors in Whole Cells}

With each cell classified as a degrader or non-degrader, we calculated the percentage of cells in each class at half hour intervals throughout the experiment (Figure 7A). In general, treatment with the FAK inhibitor tended to increase the percentage of degrading cells, while Purvalanol A decreased the percentage of degrading cells. Only eight degrading cells were detected under BB94 treatment and these were manually conformed as false positives and excluded from further analysis. These results are comparable to those observed at the single invadopodia level and again confirm the accuracy and low rate of false positives obtained using the developed automated analysis methods.

The area of ECM degraded by each cell was also quantified. To find the degraded regions of the matrix, the first and last images of the time-lapse were compared; any region where the intensity had decreased by $20 \%$ was marked as degraded. These degraded areas were assigned to each cell according to the previously defined area of influence (Figure 7B). FAK inhibitor significantly increased the area degraded by each cell (62\% increase compared to control), while PP2 and Purvalanol A each decreased the average area degraded (61\% decrease compared to control). Similarly, the rate of degradation was also quantified by dividing the total area degraded by the cell lifetime (Figure 7C). For this property we observed similar trends to the total area degraded with FAK inhibitor treated cells having a higher rate of degradation (23\% increase compared to 
294

295

\section{Discussion}

297 Understanding the process of metastasis is an as yet unresolved issue in cancer biology and 298 299 control), while in the PP2-treated cells and Purvalanol A-treated cells, there was a decrease in the degradation rate (37\% and $43 \%$ decreases compared to control). migration of cancer cells from the primary tumor is preceded by degradation of the ECM, invadopodia may play a highly significant role. However, the quantification of their dynamic behaviors has been relatively limited. The framework presented here provides a reliable approach for the quantitative analysis of invadopodia behavior in both single cells and in cell populations over time. To develop this system, we gathered time-lapse image sets of the WM2664 metastatic cancer cell line expressing LifeAct-GFP (Riedl et al. 2008) forming invadopodia over an Alexa 568-labeled ECM. Since F-actin, as labeled by LifeAct, is not a conclusive marker of invadopodia on its own, we used the images from the labeled ECM to classify each F-actin puncta as either an invadopodia or not based on changes in the ECM intensity over time. After classification of the puncta, our system calculates several dynamic invadopodia properties such as lifetime and the time taken to reach maximum degradation levels.

To complement the analysis conducted at the single invadopodia level, we also designed an automated system that follows and quantifies degradation activity at the whole cell level. This approach uses images taken at a lower magnification (20X in our experiments) to gather a representative picture of the degradation behavior of cellular populations through time. Using this system, we can begin to explore dynamic aspects of cancer cell heterogeneity at the single cell level. For instance, the percentage of cells that have degraded the matrix as well as the rate and total amount of degradation performed by each cell can be quantified. Both of these systems were tested using a set of small molecule inhibitors previously demonstrated to block or enhance invadopodia formation (Chan et al. 2009; Hoshino et al. 2012; Wang et al. 1994). Our results are supported by earlier findings but also significantly extend the amount and degree to which invadopodia and cell degradation behaviors can be quantified, all within an automated image analysis framework. 
322 Many different pharmaceuticals and their potential effects on invadopodia have been examined in 323 fixed or live cell assays in the past. These include drug treatments such as BB94, Purvalanol A,

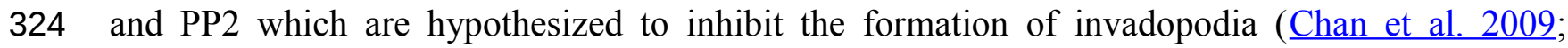
325 Hoshino et al. 2012; Wang et al. 1994). BB94 inhibits MMPs, which are the enzymatic 326 components of invadopodia that degrade the matrix (Wang et al. 1994). The effect of BB94 327 treatment is that formed puncta do not go on to degrade the matrix and are thus not formally 328 classified as invadopodia (observation of degradation is a necessary requirement for classification 329 of puncta as invadopodia) (Linder et al. 2011). Live cell imaging experiments of cancer cells 330 treated with BB94 act as means to set thresholds to minimize the number of false positives 331 detected by the system. Purvalanol A inhibits cyclin-dependent kinase which in turn inhibits the 332 tyrosine kinase Src, and PP2 is a more direct inhibitor of the tyrosine kinase Src and other 333 members of the Src family. Src is known to be involved in invadopodia formation that 334 consequently decreases when Src is inhibited by either Purvalanol A or PP2 (Chan et al. 2009; 335 Hoshino et al. 2012). On the other hand FAK Inhibitor II is hypothesized to enhance the 336 formation of invadopodia. Studies using FAK knockdown have demonstrated an increase in 337 invadopodia suggesting FAK regulates and suppresses invadopodia formation (Chan et al. 2009). 338 Therefore FAK inhibitor II, which decreases FAK autophosphorylation and activation, should 339 enhance invadopodia formation. Automated analysis as presented in the current study has shown 340 the same decrease in invadopodia formation using BB94, PP2 and Purvalanol A, as well as an 341 increase in invadopodia formation following treatment with FAK inhibitor II. Our results clearly 342 demonstrate that the software developed accurately detects known changes in invadopodia 343 formation in response to characterized perturbations.

344 A wide range of other fluorescent probes and tools are also capable of being used in the 345 automated analyses described here, such as Tks5 (Tang et al. 2013) or cortactin (Artym et al. 346 2006). Invadopodia proteins need to be fluorescently tagged and be present at invadopodia during 347 the degradation process in order to be assessed using our system. Many of these alternative tags 348 should, in fact, more reliably mark invadopodia than the LifeAct F-actin label used in this work, 349 making analysis with these markers less likely to result in false positives and decrease the 350 requirement for manual confirmation of potential false positives as required in the BB94 samples 351 in the current study. We note that the invadopodia described here were very similar to the 20-60 352 minute lifetimes characterized in a breast cancer cell line, though this may also depend on the 353 marker used (Beaty et al. 2013; Sharma et al. 2013b). The cell population analysis system can 
354 also be adapted to use alternative cell markers such as dyes or membrane associated fluorescent 355 markers and may be further improved by the addition of a nuclear marker, making it possible to 356 reliably split cell clumps using a watershed segmentation (Malpica et al. 1997). Alternative ECM 357 labeling methodologies such as dye-quenched gelatins, or other ECM substrates such as 358 fibronectin can similarly be utilized. This analysis tool is readily adaptable outside of the field of 359 cancer research, for instance, for the examination of the related invasive structure podosomes, 360 which are found in highly migratory cells such as osteoclests and macrophages (Albiges-Rizo et 361 al. 2009; Block et al. 2008).

362 The software to process the labeled puncta in single cells and in cell populations through time has 363 been released as open source packages available through the Gomez lab github repository 364 (https:/github.com/gomezlab/). In addition, the single cell analysis of individual puncta can be 365 performed through a web application (http://ias.bme.unc.edu/), which does not require the user to 366 download or install any software to process a set of invadopodia images and also allows the 367 ability to adjust thresholds to appropriate values. These two complementary analysis systems 368 allow the quantification of invadopodia behavior at the single invadopodia and single cell levels. 369 Combined with high throughput imaging methodologies, this analysis tool will be highly useful 370 in screening small molecule inhibitors for efficacy in inhibiting invadopodia formation in cancer

371 cells as well as quantifying genetic and cellular heterogeneity that may underlie related metastatic 372 behaviors.

\section{Acknowledgements}

374 We gratefully acknowledge the UNC-Olympus Imaging Research Center, UNC Research

375 Computing, as well as members of the Bear and Gomez labs for critical discussions and 376 comments on the manuscript. 


\section{References}

378 Albiges-Rizo C, Destaing O, Fourcade B, Planus E, and Block MR. 2009. Actin machinery and mechanosensitivity in invadopodia, podosomes and focal adhesions. $J$ Cell Sci 122:3037-3049.

Artym VV, Yamada KM, and Mueller SC. 2009. ECM degradation assays for analyzing local cell invasion. Methods Mol Biol 522:211-219.

Artym VV, Zhang Y, Seillier-Moiseiwitsch F, Yamada KM, and Mueller SC. 2006. Dynamic interactions of cortactin and membrane type 1 matrix metalloproteinase at invadopodia: defining the stages of invadopodia formation and function. Cancer Res 66:3034-3043.

Beaty BT, Sharma VP, Bravo-Cordero JJ, Simpson MA, Eddy RJ, Koleske AJ, and Condeelis J. 2013. $\beta 1$ integrin regulates Arg to promote invadopodial maturation and matrix degradation. Molecular Biology of the Cell 24:1661-1675.

Block MR, Badowski C, Millon-Fremillon A, Bouvard D, Bouin AP, Faurobert E, Gerber-Scokaert D, Planus E, and Albiges-Rizo C. 2008. Podosome-type adhesions and focal adhesions, so alike yet so different. Eur J Cell Biol 87:491-506.

Chan KT, Cortesio CL, and Huttenlocher A. 2009. FAK alters invadopodia and focal adhesion composition and dynamics to regulate breast cancer invasion. J Cell Biol 185:357-370.

Chen WT. 1989. Proteolytic activity of specialized surface protrusions formed at rosette contact sites of transformed cells. J Exp Zool 251:167-185.

Destaing O, Block MR, Planus E, and Albiges-Rizo C. 2011. Invadosome regulation by adhesion signaling. Curr Opin Cell Biol 23:597-606. 
Haier J, Korb T, Hotz B, Spiegel HU, and Senninger N. 2003. An intravital model to monitor steps of metastatic tumor cell adhesion within the hepatic microcirculation. J Gastrointest Surg 7:507-514; discussion 514-505.

Hoshino D, Branch KM, and Weaver AM. 2013. Signaling inputs to invadopodia and podosomes. J Cell Sci 126:2979-2989.

Hoshino D, Jourquin J, Emmons SW, Miller T, Goldgof M, Costello K, Tyson DR, Brown B, Lu Y, Prasad NK, Zhang B, Mills GB, Yarbrough WG, Quaranta V, Seiki M, and Weaver AM. 2012. Network analysis of the focal adhesion to invadopodia transition identifies a PI3KPKCalpha invasive signaling axis. Sci Signal 5:ra66.

Linder S, Wiesner C, and Himmel M. 2011. Degrading devices: invadosomes in proteolytic cell invasion. Annu Rev Cell Dev Biol 27:185-211.

Malpica N, de Solorzano CO, Vaquero JJ, Santos A, Vallcorba I, Garcia-Sagredo JM, and del Pozo F. 1997. Applying watershed algorithms to the segmentation of clustered nuclei. Cytometry 28:289-297.

Matov A, Applegate K, Kumar P, Thoma C, Krek W, Danuser G, and Wittmann T. 2010. Analysis of microtubule dynamic instability using a plus-end growth marker. Nat Methods 7:761768.

Murphy DA, and Courtneidge SA. 2011. The 'ins' and 'outs' of podosomes and invadopodia: characteristics, formation and function. Nat Rev Mol Cell Biol 12:413-426.

Riedl J, Crevenna AH, Kessenbrock K, Yu JH, Neukirchen D, Bista M, Bradke F, Jenne D, Holak TA, Werb Z, Sixt M, and Wedlich-Soldner R. 2008. Lifeact: a versatile marker to visualize F-actin. Nat Methods 5:605-607.

Sharma V, Entenberg D, and Condeelis J. 2013a. High-Resolution Live-Cell Imaging and TimeLapse Microscopy of Invadopodium Dynamics and Tracking Analysis. In: Coutts AS, ed. Adhesion Protein Protocols: Humana Press, 343-357.

Sharma Ved P, Eddy R, Entenberg D, Kai M, Gertler Frank B, and Condeelis J. 2013b. Tks5 and SHIP2 Regulate Invadopodium Maturation, but Not Initiation, in Breast Carcinoma Cells. Current Biology 23:2079-2089.

Stoletov K, Strnadel J, Zardouzian E, Momiyama M, Park FD, Kelber JA, Pizzo DP, Hoffman R, VandenBerg SR, and Klemke RL. 2013. Role of connexins in metastatic breast cancer and melanoma brain colonization. J Cell Sci 126:904-913.

Tang H, Li A, Bi J, Veltman DM, Zech T, Spence HJ, Yu X, Timpson P, Insall RH, Frame MC, and Machesky LM. 2013. Loss of Scar/WAVE complex promotes N-WASP- and FAKdependent invasion. Curr Biol 23:107-117.

Tarone G, Cirillo D, Giancotti FG, Comoglio PM, and Marchisio PC. 1985. Rous sarcoma virustransformed fibroblasts adhere primarily at discrete protrusions of the ventral membrane called podosomes. Exp Cell Res 159:141-157.

Wang X, Fu X, Brown PD, Crimmin MJ, and Hoffman RM. 1994. Matrix metalloproteinase inhibitor BB-94 (batimastat) inhibits human colon tumor growth and spread in a patientlike orthotopic model in nude mice. Cancer Res 54:4726-4728.

Wang $Y$, and McNiven MA. 2012. Invasive matrix degradation at focal adhesions occurs via protease recruitment by a FAK-p130Cas complex. J Cell Biol 196:375-385. 


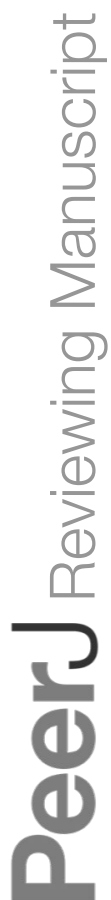

PeerJ reviewing PDF | (v2014:03:1861:1:0:NEW 3 Jun 2014) 


\section{Figure 1}

Segmentation of puncta from LifeAct-GFP images.

(A) Epifluorescence image of WM2664 cell expressing LifeAct-GFP. (B) Image from Part A passed through a high-pass filter. (C) Contour plots showing the detection errors for identification of puncta seeds. (D) Locations of puncta seeds accepted with minimum seed size 6 and high-passed threshold of 3. (E) Error rates on a pixel basis as a function of the puncta expansion threshold. (F) Puncta area plotted versus ratio between major and minor axes in puncta manually identified in either control or BB94 treated cells. (G) Locations of segmented LifeAct-GFP puncta based on seeding, expansion, area and major over minor axes filtering.
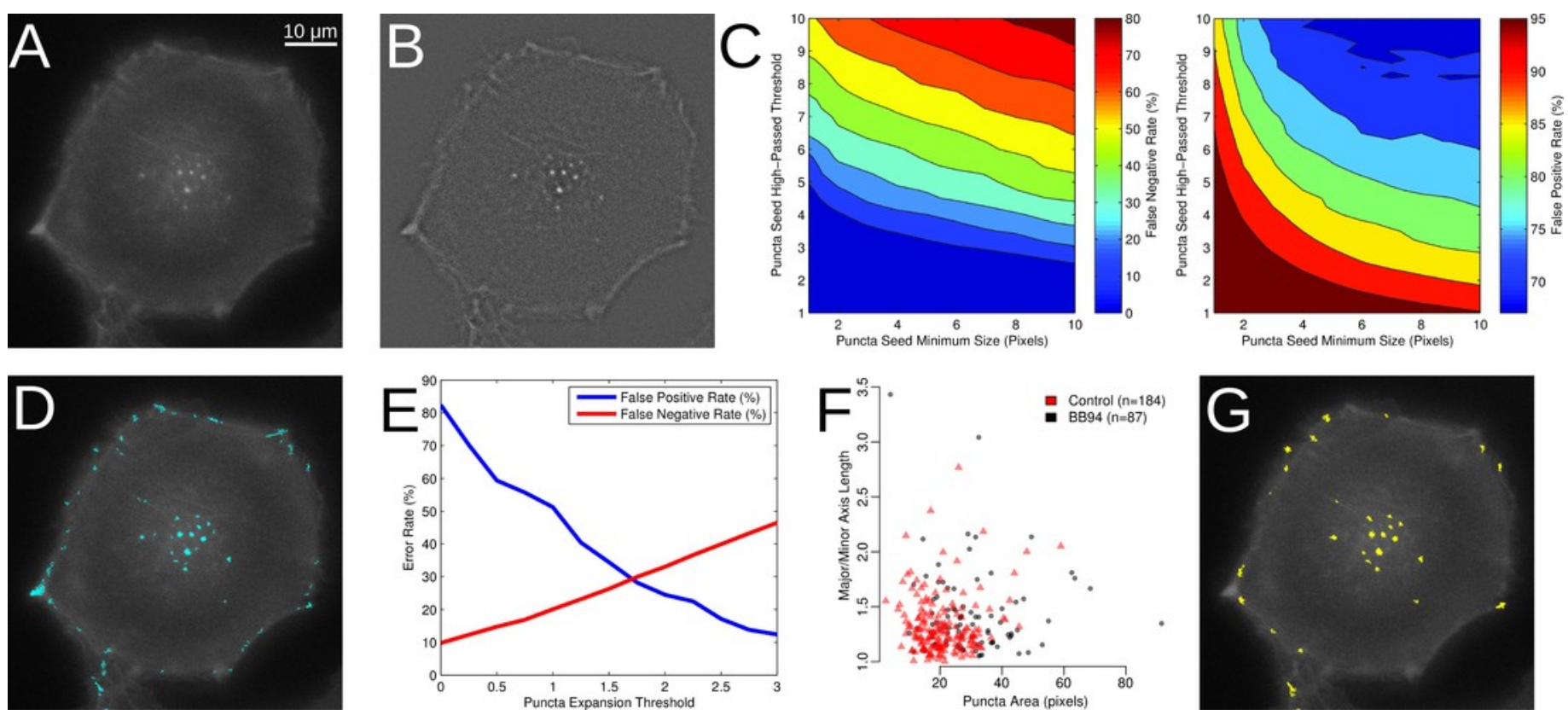


\section{Figure 2}

Properties of segmented and tracked puncta in control cells.

(A) Histogram of the lifetime of the segmented puncta. Inset graph shows the lifetime of puncta with lifetime of 12 frames or more. (B) Histogram of the average puncta area for puncta with lifetimes over $1 \mathrm{hr}$. (C) Histogram of the distance to the nearest cell edge.
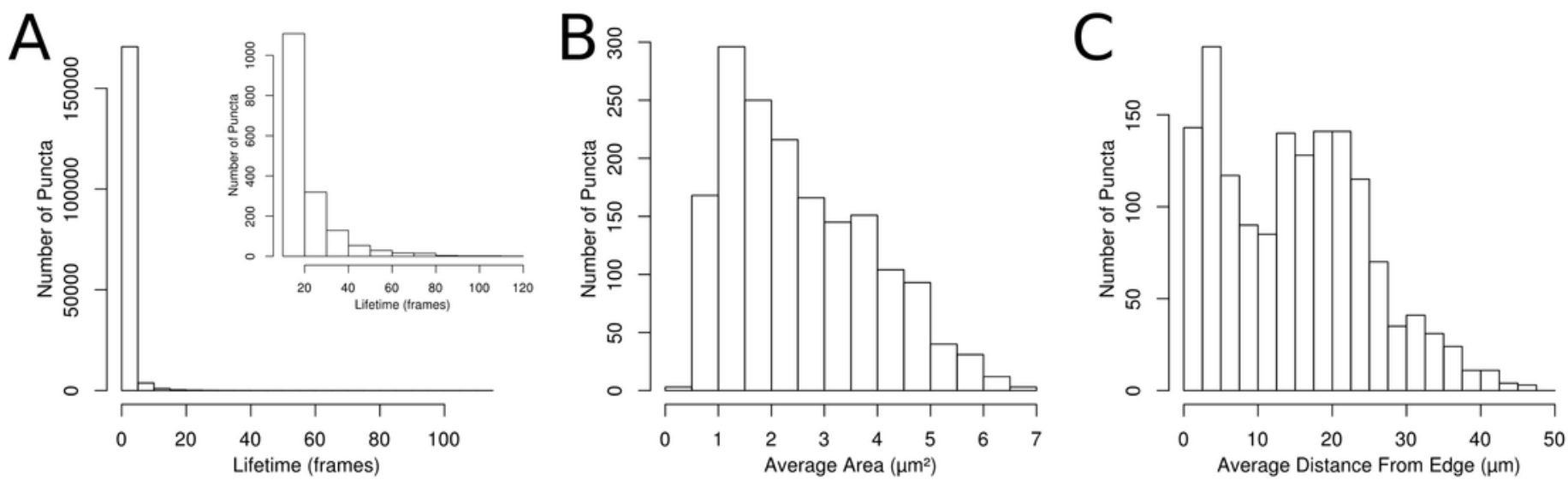


\section{Figure 3}

Measurement of ECM degradation underneath single puncta.

(A) Cartoon representation of a single puncta and the corresponding ECM underneath that degrading puncta. (B) Small multiple visualization of a single degrading puncta from a control cell and corresponding ECM intensities. The puncta is outlined in green, while the region classified as the local background is shaded purple. The first column shows the LifeAct and ECM images immediately before puncta formation and the last column shows the same areas immediately after puncta disappearance. (C) Small multiple visualization of a nondegrading puncta. (D) Boxplots of the mean local corrected difference in four of the experimental conditions. The box indicates the 25 th and 75 th percentiles, while the bold line indicates the median and the whiskers extend to 1.5 times the interquartile range. ${ }^{*}$ indicates $p<0.05$ by t-test. 

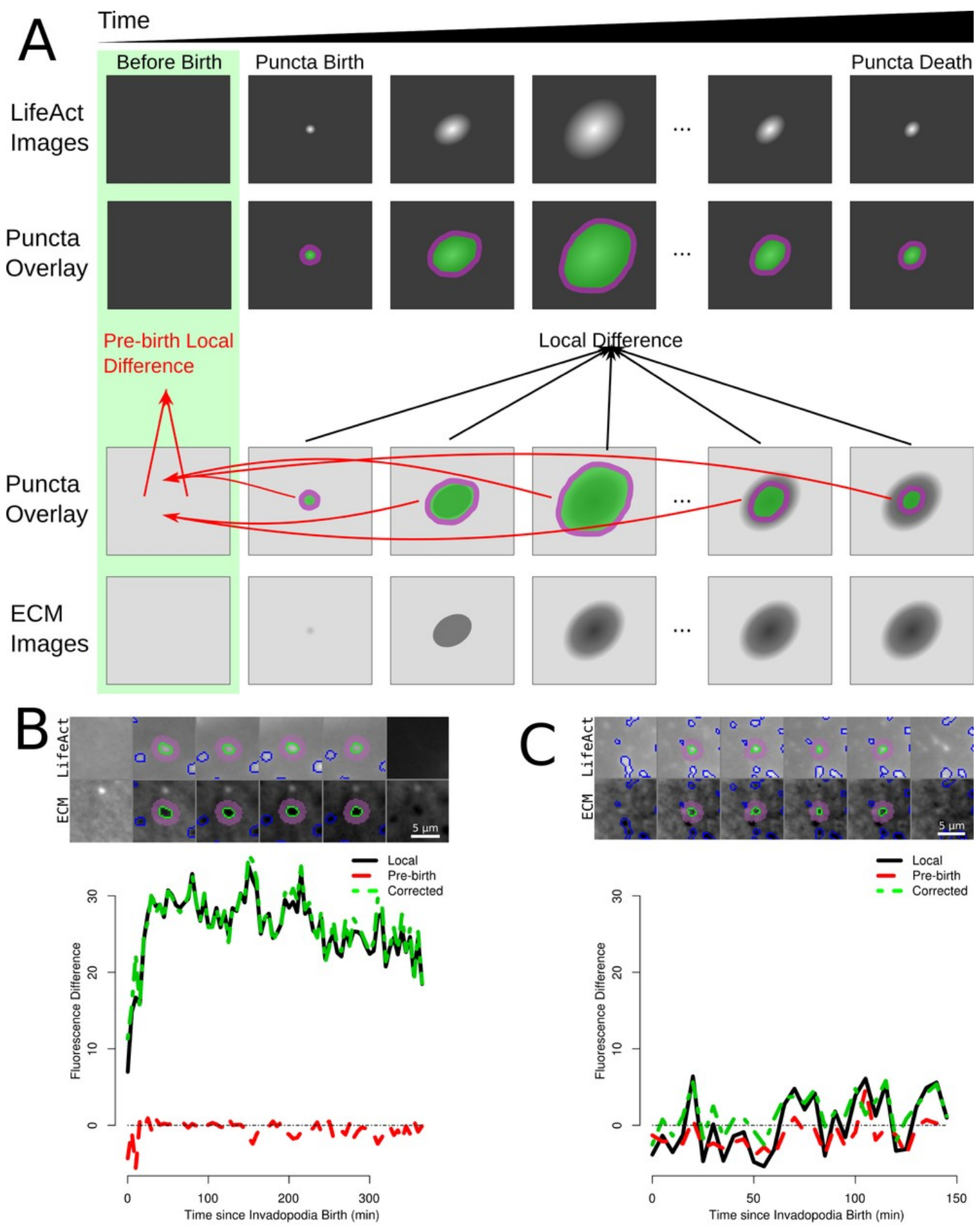

D

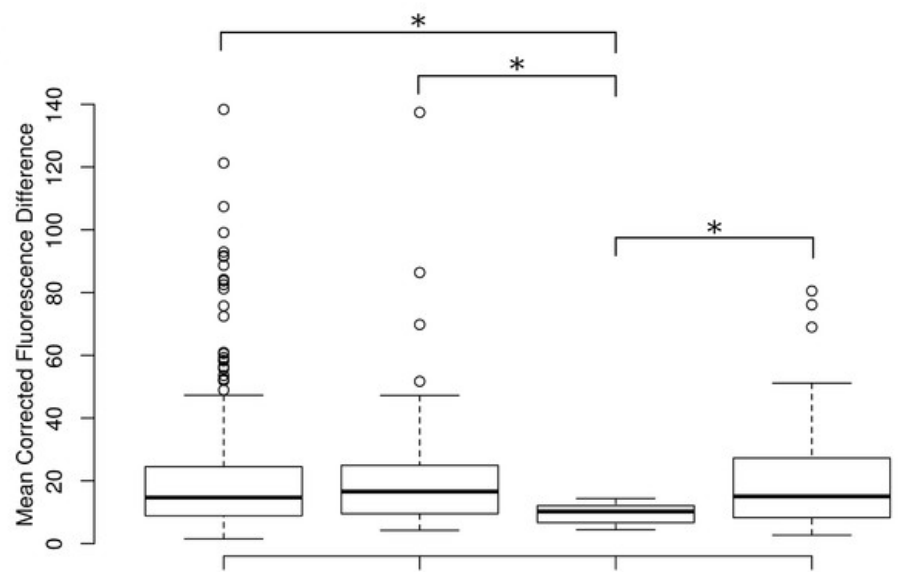

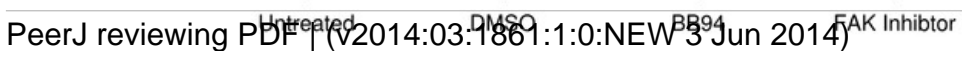




\section{Figure 4}

Properties automatically extracted from the identified invadopodia.

(A) The average area of invadopodia. (B) The average distance from the cell edge of invadopodia. (C) The lifetime of invadopodia. (D) The average time to maximum matrix degradation.

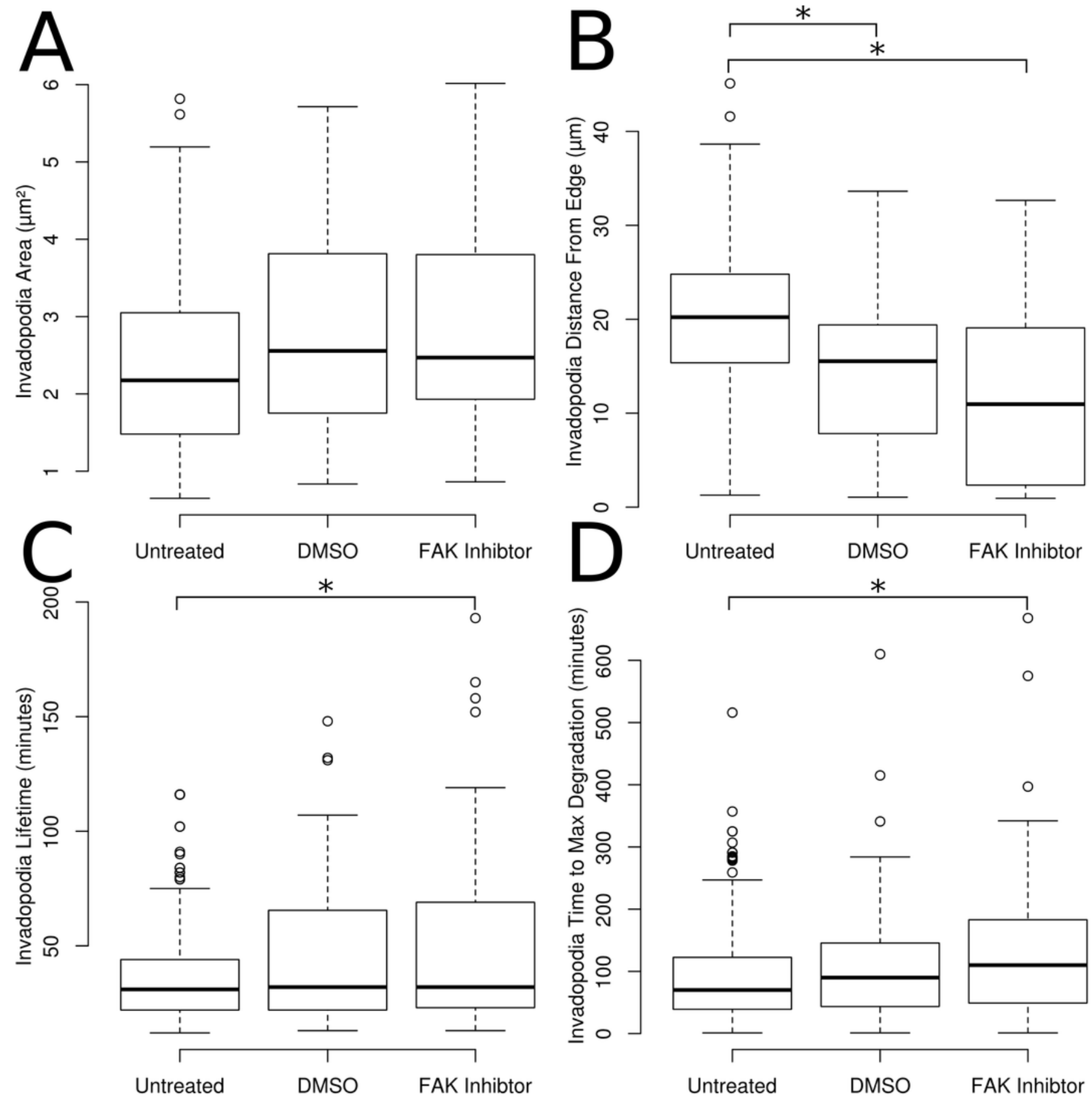




\section{Figure 5}

Identification of LifeAct-GFP expressing WM2664 cells.

(A) Sample LifeAct-GFP image from a $25 \mathrm{hr}$ time-lapse experiment after photobleaching and flat-field correction. (B) Same image as in part A, with the segmented cells outlined in yellow. (C) The distribution of object sizes detected based on intensity thresholding. (D) The distribution of the lifetime of objects detected in the control time-lapse image sets. (E) Sample fluorescent ECM image from the same time and field as in part A. (F) The ECM channel in the same field as in part $\mathrm{E}$, at the end of the $25 \mathrm{hr}$ experiment. The two sub-images show the first and last image of two cells outlined in red identified in the field. The lower portion of each sub-image shows the ECM channel immediately underneath each cell.
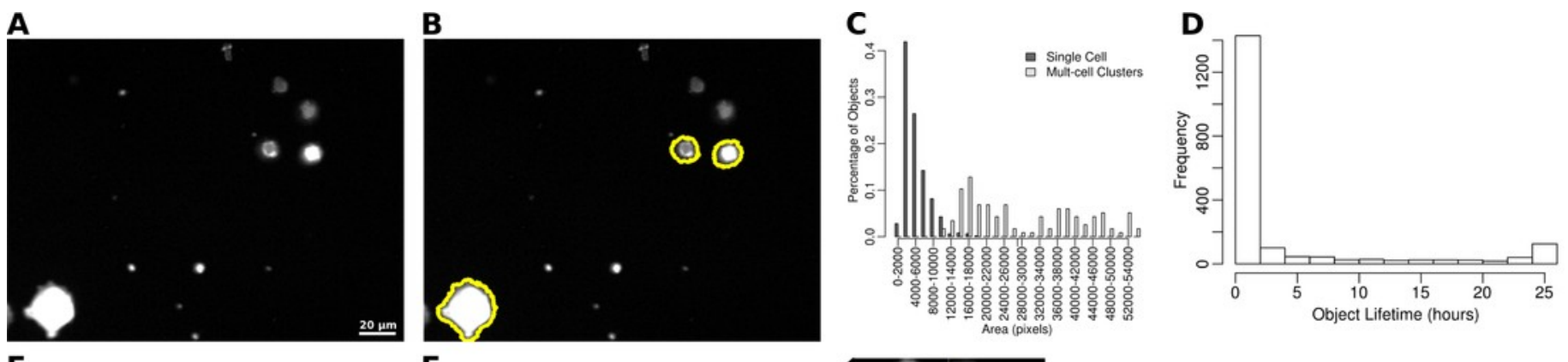

$\mathbf{E}$

$\mathbf{F}$
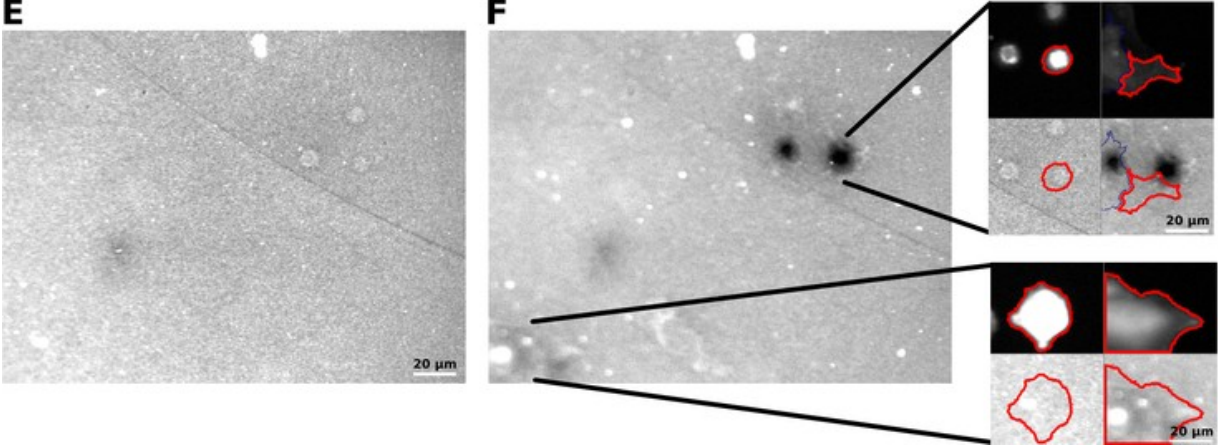


\section{Figure 6}

Determination of single cell degrader status.

(A) Cartoon representation of two cells of which one cell degrades the matrix (Cell \#1) and another non-degrading cell (Cell \#2). Also shown are the overlapping areas of the cell location and the results of comparing the first and last images from the image set. (B) Example small multiple tracks of single cells through time and the corresponding measurement of the percent of matrix degraded between each image. The colors outlining each image on the left corresponds to the same color line in the plot on the right $(C)$ Boxplots of the overall percentage of fluorescent ECM removed underneath control, DMSO treated and BB94 treated cell. ${ }^{*}$ indicate $p<0.05$ via t-test. 
A

Image \#1

Image \#2

Image \#N

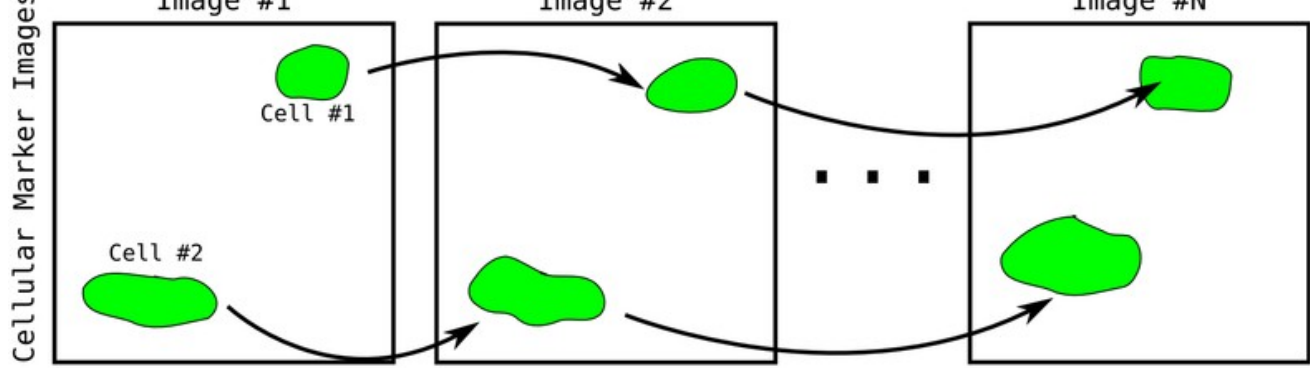

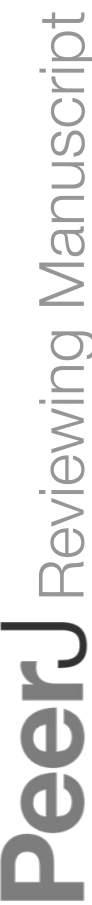
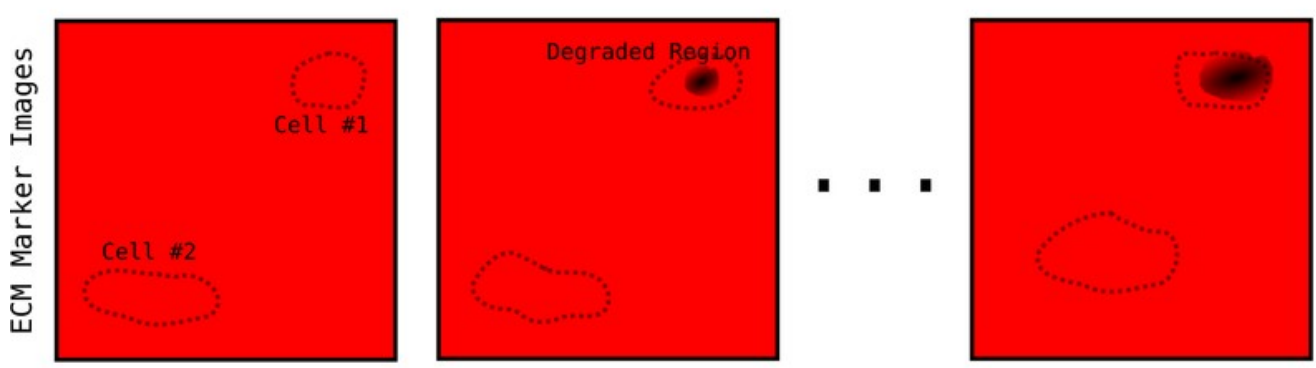

\section{B}

Time

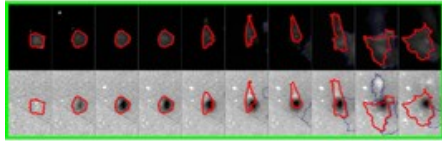

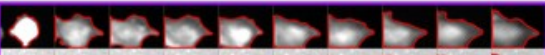

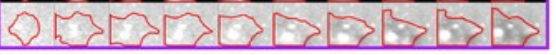

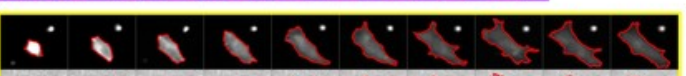

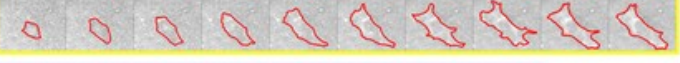

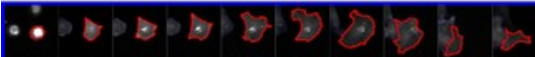

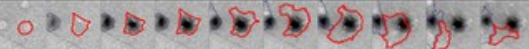

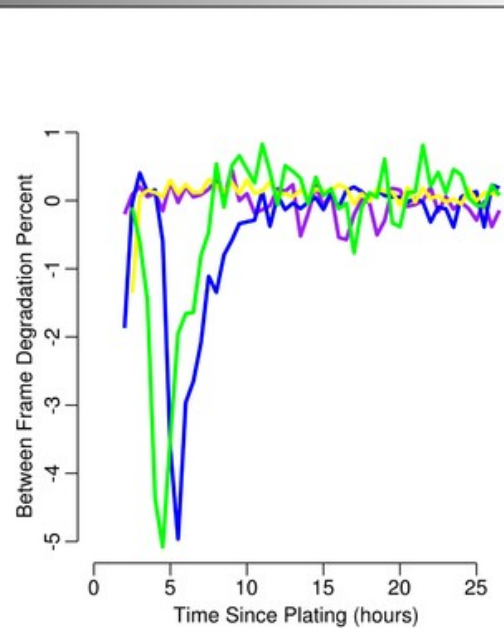

Final Comparison Images
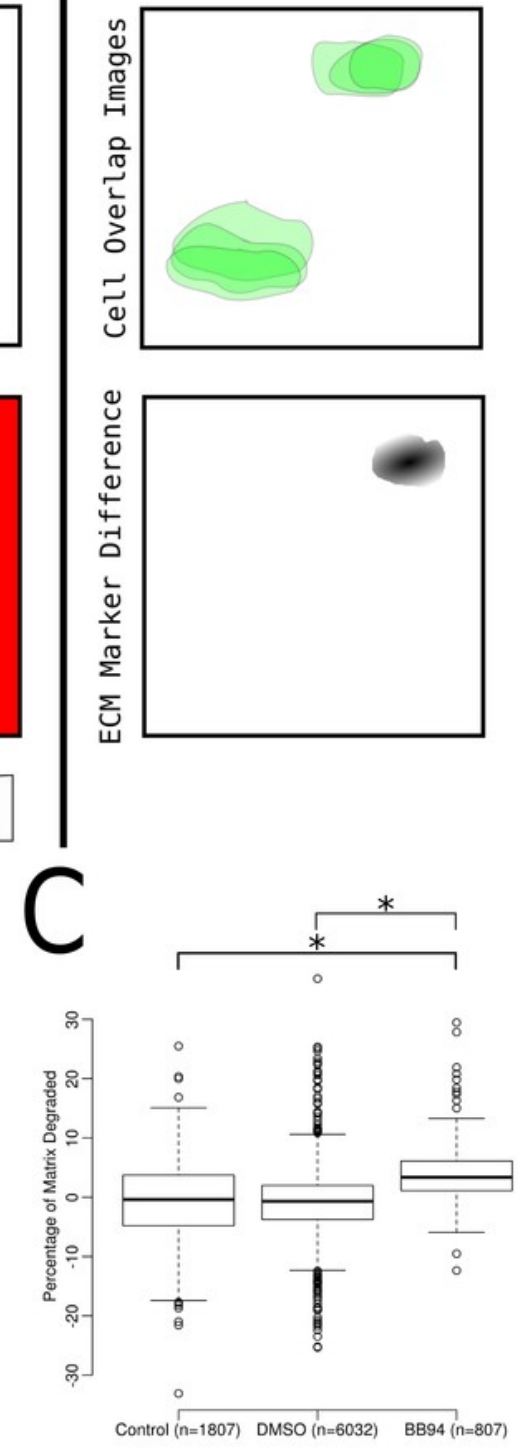


\section{Figure 7}

Properties of degrading cells.

(A) Percentage of cells classified as degraders over the $25 \mathrm{hrs}$ imaging time frame.

Percentages are averages from several experiments ( $n=5$ control, $n=2$ BB94, $n=14$ DMSO, $n=4$ PP2, $n=3$ Purvalanol $A$ and $n=4$ FAK Inhibitor). (B) The total area and (C) rate of degradation from each cell classified as an invader, ${ }^{*} p<0.05$ via t-test.
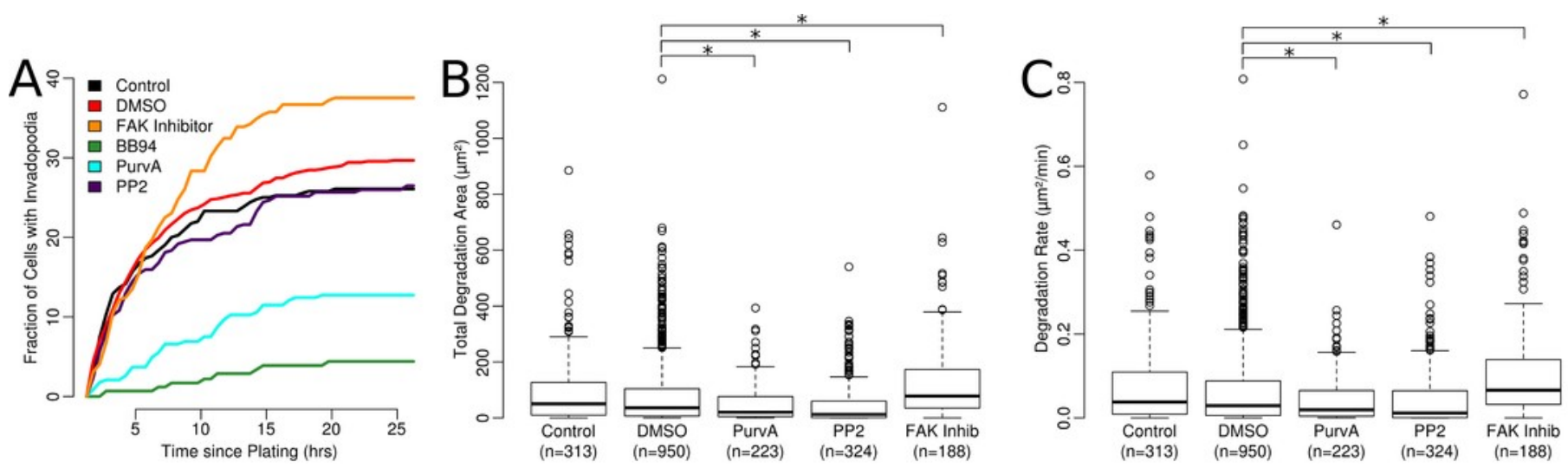